\title{
Proverb Comprehension in Individuals with Agenesis of the Corpus Callosum
}

Jamie L. Rehmela, Warren S. Brown ${ }^{\mathrm{a}}$, and Lynn K. Paula,b

aFuller Graduate School of Psychology, Travis Research Institute, 180 N. Oakland

Ave., Pasadena, CA 91101 USA

${ }^{\mathrm{b} C a l i f o r n i a}$ Institute of Technology, Division of Humanities and Social Sciences, MC

228-77, 1200 E. California Blvd., Pasadena, CA 91125 USA

Corresponding Authors:

Lynn K. Paul

Baxter MC 228-77

California Institute of Technology

1200 E California Blvd

Pasadena CA 91125

Email: lkpaul@hss.caltech.edu

626-395-4077

Warren S. Brown

Fuller Graduate School of Psychology

180 N. Oakland Ave.

Pasadena, CA 91101

Email: wsbrown@fuller.edu

626-584-5525-office 


\begin{abstract}
Comprehension of non-literal language involves multiple neural systems likely involving callosal connections. We describe proverb comprehension impairments in individuals with isolated agenesis of the corpus callosum (AgCC) and normal-range general intelligence. Experiment 1 compared Gorham Proverb Test (Gorham, 1956) performance in 19 adults with AgCC and 33 neurotypical control participants of similar age, sex, and intelligence. Experiment 2 used the Proverbs subtest of the Delis-Kaplan Executive Function System (D-KEFS, 2001) to compare 19 adults with AgCC and 17 control participants with similar age, sex, and intelligence. Gorham Proverbs performance was impaired in the AgCC group for both the free-response and multiple-choice tasks. On the D-KEFS proverbs test, the AgCC group performed significantly worse on the free-response task (and all derivative scores) despite normal levels of performance on the multiple-choice task. Covarying verbal intelligence did not alter these outcomes. However, covarying a measure of non-literal language comprehension considerably reduced group differences in proverb comprehension on the Gorham test, but had little effect on the D-KEFS group differences. The difference between groups seemed to be greatest when participants had to generate their own interpretation (free response), or in the multiple choice format when the test included many proverbs that were likely to be less familiar. Taken together, the results of this study clearly show that proverb comprehension is diminished in individuals with AgCC compared to their peers.
\end{abstract}


Keywords: proverbs, corpus callosum, non-literal language, callosal agenesis 


\section{Introduction}

Exploration of the cognitive deficits associated with malformations of the corpus callosum has proven fruitful in understanding the role of the corpus callosum in human cognition (Paul, 2011; Paul et al., 2007). While individuals with agenesis of the corpus callosum (AgCC) can have basic intelligence within or above the normal range, they nevertheless have a consistent pattern of more subtle cognitive challenges, including difficulty understanding non-literal expressions in language (Brown, Symingtion, VanLancker-Sidtis, Dietrich, \& Paul, 2005; Paul, Van Lancker-Sidtis, Schieffer, Dietrich, \& Brown, 2003) and verbal humor (Brown, Paul, Symington, \& Dietrich, 2005). These deficits in humor and non-literal expression comprehension have primarily been identified using multiple-choice measures. The question remains whether individuals with AgCC have even greater difficulty understanding metaphoric meanings of language in a natural context, i.e. when alternative interpretations are not available from which to choose.

A previous small-group study of 10 persons with AgCC demonstrated difficulty with comprehension of non-literal expressions in the Gorham Proverbs test (Paul et al., 2003). However, the relationship between recognition of proverb meaning among several alternatives (multiple choice responses) and generation of an appropriate meaning (free responses) was unclear in this study. We had expected greater difficulty in generation compared to recognition of proverb meaning since generation would require unaided construction of an appropriate semantic field that expresses the metaphoric, second-order relationships in the proverb. Although there was some support for this hypothesis in the group means for the multiple 
choice and free response subtests, there was not a significant group by responsetype interaction in this study. The current study more thoroughly addresses the issue of proverb interpretation in individuals with AgCC, particularly with respect to recognition versus generation of proverb meaning, by examining this question using two different tests of proverb comprehension and a much larger group of participants.

\subsection{Agenesis of the Corpus Callosum}

AgCC is a congenital disorder in which axons that typically connect the two cerebral hemispheres do not cross the midline of the brain, and thus the corpus callosum is completely or partially absent. In most cases of AgCC, the anterior commissure is present and therefore provides some inter-hemispheric connectivity (Rauch \& Jinkins, 1994). AgCC is often associated with mental retardation and with other neurological syndromes, but it may also occur in isolation and in individuals with average or above average general intelligence (Chiarello, 1980; Sauerwein, Nolin, \& Lassonde, 1994). By studying the sub-population of individuals with isolated AgCC and intelligence quotient (IQ) at or above normal range, we are gaining insights into the role of the corpus callosum in development and maintenance of higher order cognition and psychosocial skills.

Individuals with isolated $\mathrm{AgCC}$ and intact IQ appear to have a consistent profile of mild cognitive and developmental deficits. This profile includes mild to moderate difficulties on tasks necessitating bimanual coordination of motor movements (Jeeves, Silver, \& Jacobson, 1988; Jeeves, Silver, \& Milner, 1988; Mueller, Marion, Paul, \& Brown, 2009), diminished interhemispheric transfer of complex 
sensory information (Brown, Jeeves, Dietrich, \& Burnison, 1999; Imamura, Yamadori, Shiga, Sahara, \& Abiko, 1994; Jeeves, 1979; Jeeves \& Silver, 1988; Karnath, Schumacher, \& Wallesch, 1991; Sauerwein \& Lassonde, 1983) and poor complex novel problem-solving (Gott \& Saul, 1978; Sauerwein et al., 1994; Smith, Rourke, \& Rourke, 1994; Solursh, Margulies, Ashem, \& Stasiak, 1965). On tasks involving more complex cognitive processes, performance is typically characterized by slow reaction times and processing speed (Brown et al., 1999; Brown, Thrasher, \& Paul, 2001; Hines, Paul, \& Brown, 2002; Marco et al., 2012). Preliminary evidence also suggests problems in the encoding of complex verbal memories (Erickson, Paul, \& Brown, 2014). Socially, individuals with AgCC exhibit a limited theory of mind (Symington, Paul, Symington, Ono, \& Brown, 2010) and difficulties interpreting interpersonal relations (W. Brown \& L. Paul, 2000; L. Paul, 2004; Turk, Brown, Symingtion, \& Paul, 2010).

It has been suggested that AgCC involves a core cognitive deficit in the speed and capacity for time-pressured, complex, novel problem-solving (Brown \& Paul, 2000; Marco et al., 2012; Paul et al., 2007). Persons with AgCC appear to have limited cognitive resources available for any given task such that relatively more complex and novel tasks manifest greater impairment. This is likely due to the reduced size and complexity of cortical networks that can be marshaled for cognitive tasks when the corpus callosum is absent.

\subsection{Proverbs and Non-literal Language in AgCC}

A proverb has been defined as "a short, generally know sentence of the folk which contains wisdom, truth, morals, and traditional views in a metaphorical, fixed, 
and memorizable form and which is handed down from generation to generation" (Mieder, 1993, p. 24). While proverbs are complete sentences, idioms are not and generally do not make literal sense. However, the metaphorical nature of proverbs means that they are, like idioms, language expressions in which comprehension requires the hearer/reader to map the causal structure of the proverb from the domain of its literal meaning (e.g., "Rome was not built in a day.") onto the current context. The hearer/reader must grasp the basic elements of the proverb and metaphorically map these elements onto the current situation to understand the semantic intent of the proverb. Previous studies involving the comprehension of non-literal language (idioms) and humor in individuals with AgCC have suggested that one important domain in which the core deficits in complex, novel problemsolving are manifest is in the comprehension of second-order (non-literal) meaning in language (Brown, Paul, et al., 2005; Brown, Symingtion, et al., 2005; Paul et al., 2003). Adequate proverb understanding should also require similar capacity for comprehension of second-order meaning in language.

In case studies of two individuals with AgCC, Brown and Paul (Brown \& Paul, 2000) administered the Gorham's proverbs task. Neither subject was able to produce appropriate answers on the free-response format of Gorham's proverbs, but both achieved a low average abstraction score on the multiple-choice format. We hypothesized that their performance was stronger on multiple choice because selection between alternate interpretations is a fundamentally less complex task than free response, which requires spontaneous generation of proverb interpretation, as well as generation of a verbal response. However, in a follow-up 
study of 10 individuals with AgCC (including the 2 previously described) and 14 matched controls, the AgCC group performed more poorly than controls on both the free-response and multiple-choice proverb comprehension tasks. While there was some indication of greater difficulty with the free response format, the interaction effect between group and response format was not significant leaving some ambiguity about the degree to which AgCC impacts proverb interpretation when given choices among options (Paul et al., 2003). In the expanded study, responses given in the free response format were also analyzed for differences in response length, correct form, and correct content. Individuals with AgCC produced shorter responses, and were more likely to have both incorrect form (e.g., not matching elements in the proverb with elements in their responses) and incorrect content (i.e., responses did not adequately represent the conventional meaning).

In this same paper it was also shown that individuals with AgCC performed significantly worse than controls when asked to select one out of four line drawings which best fits with a non-literal statement (common idioms) read aloud by the examiner. On this task, the Familiar and Novel Comprehension Test (FANL-C) (Kempler \& Van Lancker-Sidtis, 1996), the AgCC group performed similarly to controls when selecting images to match literal statements. Since this pattern of performance in the AgCC group was similar to that of persons with righthemisphere brain damage (Van Lanker Sidtis \& Postman, 2006), the authors suggested that deficits in AgCC may reflect reduced integration of right-hemisphere and left-hemisphere semantic processes.

Some have suggested that understanding humor, like proverb 
comprehension, requires inter-hemispheric activity - that is, the right hemisphere is responsible for detecting semantic incongruence and must interact with the primary language center of the left hemisphere in order to re-establish language coherence and appreciate the underlying humor (Bihrle, Brownell, Powelson, \& Gardner, 1986; Coulson \& Williams, 2005). Consistent with this hypothesis, 16 adults with AgCC performed more poorly overall than a matched control group on a multiple-choice format test of narrative humor (Brown, Paul, et al., 2005). Incorrect selections by AgCC participants were most often straight-forward conclusions to the narrative. Importantly, the group difference on narrative humor appeared to share large portions of variance with performance on the multiple-choice section of Gorham's Proverbs Test and on the non-literal items subscale of the FANL-C, such that covarying either of these measures eliminated group differences in humor. Based on the shared variance among these tasks, it was concluded that persons with AgCC have a general deficit in comprehension of second-order meaning in language that is common to tests of proverb, non-literal language, and humor comprehension.

\subsection{The Neuropsychology of Proverb Comprehension}

The comprehension of proverbs is a highly complex cognitive operation that involves a number of capacities. Thus, studies have suggested that reading proficiency (Nippold, Allen, \& Kirsch, 2001), noun comprehension (Nippold, Allen, \& Kirsch, 2000), working memory (Moran, Nippold, \& Gillon, 2006; Uekermann, Thoma, \& Daum, 2008), analogical reasoning (Nippold \& Sullivan, 1987), theory of mind capacity (Brune \& Bodenstein, 2005), and age and education (Nippold, Uhden, \& Schwarz, 1997; Uekermann et al., 2008) all play a role in understanding proverbs. 
Proverb comprehension improves through development into an individual's third decade (i.e. the 20 's) and then remains relatively consistent into the seventh (i.e. the 60's) when it begins to decline (Nippold et al., 1997).

Interpretation of proverbs is believed to be heavily reliant on executive function (Delis, Kaplan, \& Kramer, 2001; Kaiser et al., 2013; Kiang et al., 2007; McDonald, Delis, Kramer, Tecoma, \& Iragui, 2008; Murphy et al., 2013; Roca et al., 2010). The basic cognitive skills involved in non-literal language comprehension include set-shifting, cognitive inhibition, attention, and working memory, all of which are considered implicated in the executive control of cognitive processes. Working memory specifically predicts performance on tests of proverb comprehension in traumatic brain injury (Moran et al., 2006) and aging (Uekermann et al., 2008). Thus, proverbs tests have been used to assess executive function in individuals with conditions such as traumatic brain injury (Murphy et al., 2013; Yang, Fuller, Khodaparast, \& Krawczyk, 2010), schizophrenia (Kiang et al., 2007), or fronto-temporal dementia and Alzheimer's disease (Kaiser et al., 2013). Lesion studies indicate that medial-frontal brain regions are critical for proverb comprehension, as this skill is worse in individuals with medial-frontal brain damage than individuals with lesions to the lateral cortex of either the right or left hemispheres (Murphy et al., 2013).

Individuals who have suffered a traumatic brain injury also struggle to adequately interpret non-literal language and proverbs (e.g., (Moran et al., 2006; Murphy et al., 2013; Yang et al., 2010). These conditions are associated with reduced myelin and axonal integrity, particularly in the anterior and posterior 
corpus callosum (e.g., as shown by diffusion tensor imaging; Wilde et al., 2006). Impaired proverb interpretation following traumatic brain injury suggests the importance of white matter integrity and cortical interconnectivity for this skill.

\subsection{Rationale and Hypotheses}

To better clarify the importance of callosal connections in supporting proverb interpretation (both generation and recognition of correct interpretations), we present two studies of proverb comprehension in persons with AgCC and matched controls. The first study directly replicated the Paul et al. (Paul et al., 2003) study using the Gorham's Proverb Test but with a larger sample. The second experiment used the Proverb Test from the Delis-Kaplan Executive Function System (D-KEFS) to determine if the pattern of proverb comprehension in AgCC is robust to instrument variation. Based on our previous work, we predicted that the individuals with AgCC would perform more poorly than the control group on both the Gorham and D-KEFS proverbs tasks, and that the deficits in AgCC would be particularly apparent on the free-response versions of both tests where participants must generate the meaning rather than recognize and choose the best meaning from options provided. With respect to the subscales available on the D-KEFS proverbs task, it was predicted that individuals with AgCC would show deficits in both abstraction and accuracy, and that they would have particular difficulty with uncommon proverbs.

\section{Material and Methods}

For both experiments, persons with AgCC were recruited through the AgCC 
Network and the National Organization of Disorders of the Corpus Callosum or were self-referrals. Control participants for both experiments were recruited from responders to an advertisement on Craigslist.com and were included based on an initial telephone screening. AgCC and control participants were excluded if they had an FSIQ below 80, a history of head trauma, three or more seizures, major neurological conditions (e.g., stroke, Parkinson's disease, Alzheimer's disease), a psychiatric diagnosis of schizophrenia or bipolar disorder, or history of drug abuse. Potential control participants were screened to determine if they met exclusionary criteria via a telephone interview and administration of the vocabulary subtest of the WAIS-III (Wechsler, 1997).

All participants were given a current age-appropriate Wechsler intelligence test. This test was used as an exclusionary criterion. In addition, the Verbal Comprehension Index (VCI) of this test was used as a covariate to further remove between-participant variance in verbal intelligence. The VCI is an index score generated from the Wechsler scales of intelligence. In the Wechsler Adult Intelligence Scale (versions 3 and 4) VCI is generated from performance on tests of expressive vocabulary (Vocabulary), general knowledge commonly learned in school (Information) and a test of abstract verbal reasoning (Similarities). In the Wechsler Abbreviated Scales of Intelligence (second edition), VCI is based on Vocabulary and Similarities performance. We elected to use VCI instead of VIQ because VIQ is more likely to be influenced by potential confounds related to working memory (i.e. the subtests included in VCI are a subset of those included in VIQ). Handedness was assessed using a 10-item Edinburgh Handedness 
Questionnaire (Oldfield, 1971) and right-handedness was indicated by a score of 40 or greater. Finally, the FANL-C (Kempler \& Van Lancker-Sidtis, 1996) was given to all participants and used as a covariate to reveal the contributions of both literal and non-literal language comprehension. For the FANL-C, participants were read 20 literal and 20 nonliteral sentences and were required to select which line-drawing out of 4 options best represents the meaning of the sentence. This task was chosen based on previous research showing a large amount of shared variance between humor comprehension (another form of non-literal language comprehension) and the non-literal items of the FANL-C (Brown, Paul, et al., 2005).

Test results were gathered as part of a larger battery utilized to investigate the cognitive and psychosocial consequences of agenesis of the corpus callosum. Each participant read and signed a consent form describing the research. Methods for this study were approved by the Institutional Review Board of the Travis Research Institute.

\subsection{Experiment 1: Gorham's Proverbs}

2.1.1 Participants: This experiment involved 19 adults and adolescents with AgCC (15 with complete AgCC and 4 with partial AgCC) and 33 neurotypical controls. Of the participants used in this experiment, 10 of the AgCC group and 14 of the controls were used in a previous study of nonliteral language and affective prosody (Paul et al., 2003). Demographic information for participants in Experiment 1 can be found in Table 1. There were no significant differences between groups for age, $t(50)=-$ $0.57, p=.57$; full scale IQ (FSIQ) $t(50)=-0.24, p=.81$; verbal comprehension index (VCI) $t(50)=-1.15, p=.26$; or gender $X^{2}(50)=1.48, p=.22$. There was, however, a 
group difference observed for handedness, $X^{2}(50)=6.75, p=.03$, with greater number of non-right-handers in the AgCC group.

Table 1

Experiment 1: Demographic Data and Covariates by Groups for Gorham's Proverbs

\begin{tabular}{lllllll}
\hline & \multicolumn{2}{l}{ AgCC $(\mathrm{n}=19)$} & \multicolumn{3}{c}{ Control $(\mathrm{n}=33)$} \\
& $X$ & $S D$ & Range & $X$ & $S D$ & Range \\
\hline Age & 25.89 & 9.56 & $16-55$ & 24.48 & 7.95 & $17-51$ \\
FSIQ & 98.26 & 13.25 & $83-131$ & 97.58 & 7.12 & $84-111$ \\
VCI & 100.05 & 18.93 & $67-136$ & 95.79 & 7.68 & $84-112$ \\
& & & & & & \\
Literal & 19.21 & 1.08 & $16-20$ & 19.24 & 0.79 & $17-20$ \\
Non-literal** & 15.68 & 4.11 & $6-20$ & 18.73 & 1.84 & $13-20$ \\
\hline Gender (\% male) & $79 \%$ & & & $91 \%$ & & \\
Handed (\% RH) & $68 \%$ & & & $94 \%$ & & \\
\hline
\end{tabular}

Note. AgCC = participants with agenesis of corpus callosum; FSIQ = full scale intelligence quotient; $\mathrm{VCI}=$ verbal comprehension index; Non-literal and Literal refer to subscales of the Familiar and Novel Language Comprehension task; ${ }^{* *} \mathrm{p}<$ .01 .

2.1.1 Procedure: The 12-item free-response and 40-item multiple-choice formats of the Gorham's Proverbs Test (Gorham, 1956) were used to assess proverb interpretation and comprehension. In the free-response format, participants were asked to read and write out an interpretation of each of 12 different proverbs. On the multiple-choice format, participants were asked to read 40 proverbs (including the original 12) and choose one of four proverb interpretations, with one choice being the most accurate and abstract while the others were inaccurate and/or concrete. Answers were scored according to Gorham's criteria.

All analyses were conducted with raw scores using age as a covariate. In 
order to explore the relationship between proverb comprehension and verbal intelligence, the VCI was employed as a covariate in post-hoc analyses. Similarly, the literal and non-literal item subscales of the FANL-C were also used as covariates in post-hoc analyses.

\subsection{Experiment 2: D-KEFS Proverbs}

2.2.1 Participants: Experiment 2 involved 19 adults and adolescents with AgCC (15 with complete and 4 with partial $\mathrm{AgCC}$ ) and 17 neurotypical controls. Two participants with AgCC were included in both Experiment 1 and 2. Demographic information for participants in Experiment 2 can be found in Table 2. There were no significant differences between groups for age, $t(34)=1.28, p=.21$; FSIQ $t(34)=$ $1.57, p=.13$; handedness, $X^{2}(34)=0.29, p=.87$; or gender $X^{2}(34)=0.00, p=.99$. The AgCC group did however have lower VCI performance, $t(34)=2.18, p=.04$.

Table 2

Experiment 2: Demographic Data and Covariates by Groups for D-KEFS

\begin{tabular}{lllllll}
\hline & \multicolumn{2}{c}{ AgCC $(\mathrm{n}=19)$} & \multicolumn{3}{c}{ Control $(\mathrm{n}=17)$} \\
& $X$ & $S D$ & Range & $X$ & $S D$ & Range \\
\hline Age & 25.47 & 10.52 & $16-52$ & 29.47 & 7.22 & $20-45$ \\
FSIQ & 95.68 & 13.27 & $80-129$ & 102.00 & 10.53 & $84-114$ \\
VCI* & 99.16 & 12.76 & $82-129$ & 107.76 & 10.63 & $91-126$ \\
Literal & 18.85 & 1.60 & $14-20$ & 19.12 & 0.79 & $18-20$ \\
Non-literal** & 17.40 & 3.35 & $7-20$ & 18.88 & 1.30 & $15-20$ \\
\hline Gender (\% male) & $53 \%$ & & & $53 \%$ & & \\
Handed (\% RH) & $63 \%$ & & & $71 \%$ & & \\
\hline
\end{tabular}

Note. AgCC = participants with agenesis of corpus callosum; FSIQ = full scale intelligence quotient; $\mathrm{VCI}$ = verbal comprehension index; Non-literal and Literal refer to subscales of the Familiar and Novel Language Comprehension task; ${ }^{*} \mathrm{p}<$ .05 ; $^{* *} \mathrm{p}=.05$ (one-tailed). 
2.2.2 Procedure: Participants were given the D-KEFS (Delis et al., 2001) in its entirety, of which the proverbs subtest was used in this study. Participants completed both the free-response and multiple-choice subscales of this test. In the free-response section, participants were read aloud eight proverbs, and then, after each proverb, were asked to provide their best interpretation, which was written down verbatim by the test administrator. The free-response task provides a total score and four sub-scores: accuracy and abstraction, as well as accuracy for common and for uncommon proverbs. The abstraction score is based on how abstract the response is independent of interpretative accuracy. Conversely, accuracy is based on the accuracy of the interpretation, independent of how abstract the response is.

For the multiple-choice task, the administrator read the same eight proverbs one at a time and participants selected an answer from four written options. The multiple-choice subscale provides a total score and four sub-scores: number of correct abstract responses, number of correct concrete responses, as well as accuracy for common and for uncommon proverbs.

All analyses were conducted with raw scores using age as a covariate. Given the reliance on verbal abilities for interpreting proverbs, $\mathrm{VCI}$ was used as a covariate in post-hoc analyses. Lastly, as with Experiment 1, the literal and non-literal item subscales of the FANL-C were also used as covariates.

\section{Results}

\subsection{Experiment 1: Gorham's Proverbs}


Descriptive statistics for all Gorham's measures for each group are presented in Table 3, and results of univariate ANCOVAs (covarying age) comparing groups are presented in Table 4.

Table 3

Data Summaries: Raw Score Means, Standard Deviations and Ranges for MultipleChoice \& Free Response Proverbs Tests.

\begin{tabular}{|c|c|c|c|c|c|c|}
\hline & $X$ & $S D$ & Range & $X$ & $S D$ & Range \\
\hline Gorham & \multicolumn{3}{|c|}{$\operatorname{AgCC}(n=19)$} & \multicolumn{3}{|c|}{ Control (n = 33) } \\
\hline $\begin{array}{l}\text { Free } \\
\text { Response** }\end{array}$ & 6.58 & 6.90 & $0-22$ & 10.76 & 3.99 & $5-19$ \\
\hline Multiple Choice* & 21.58 & 7.34 & $8-34$ & 26.03 & 4.67 & $17-35$ \\
\hline D-KEFS & \multicolumn{3}{|c|}{$\operatorname{AgCC}(n=19)$} & \multicolumn{3}{|c|}{ Control $(\mathrm{n}=17)$} \\
\hline $\begin{array}{l}\text { Free } \\
\text { Response** }\end{array}$ & 14.74 & 5.53 & $4-23$ & 23.24 & 5.07 & $15-31$ \\
\hline Accuracy** & 6.95 & 2.93 & $2-12$ & 11.35 & 2.21 & 8-15 \\
\hline Abstraction** & 8.74 & 3.69 & $2-16$ & 12.24 & 3.23 & $6-16$ \\
\hline a Common & 0.51 & 0.19 & $0.2-0.8$ & 0.76 & 0.16 & $0.45-0.95$ \\
\hline bUncommon & 0.39 & 0.23 & $0.0-0.92$ & 0.67 & 0.25 & $0.17-1.0$ \\
\hline Multiple Choice & 29.89 & 3.09 & $24-32$ & 31.06 & 1.75 & $26-32$ \\
\hline Abstraction & 7.21 & 1.23 & $4-8$ & 7.65 & 0.61 & $6-8$ \\
\hline Concrete & 5.58 & 3.37 & $0-8$ & 5.88 & 3.39 & $0-8$ \\
\hline aCommon & 0.94 & 0.12 & $0.6-1.0$ & 0.98 & 0.05 & $0.9-1.0$ \\
\hline bUncommon & 0.93 & 0.13 & $0.67-1.0$ & 0.96 & 0.11 & $0.67-1.0$ \\
\hline
\end{tabular}

Note. AgCC = participants with agenesis of corpus callosum.

${ }^{*} \mathrm{p}<.05 ;{ }^{* *} \mathrm{p}<.01$; corrected for multiple comparisons.

arepresents the percentage of total possible points obtained on common proverbs; brepresents the percentage of total possible points obtained on uncommon proverbs

As hypothesized, the AgCC group provided poorer quality interpretations of proverbs on the free-response task relative to the control group. However, in 
contrast to our hypothesis, the AgCC group also performed more poorly than the control group on the multiple-choice task.

Table 4

Univariate ANCOVA Results from Gorham and D-KEFS Proverbs with and without other covariates (but always covarying age)

\begin{tabular}{|c|c|c|c|c|c|c|}
\hline & $F$ & $p$ & $\eta^{2} p$ & $V C I \eta^{2} p$ & $\begin{array}{c}\text { FANL-C } \\
\text { literal } \\
\eta^{2} p\end{array}$ & $\begin{array}{c}F A N L-C \\
\text { nonliteral } \\
\eta^{2} p\end{array}$ \\
\hline Gorham & $d f(1,49)$ & & & & & \\
\hline Free Response & 7.61 & .008 & .13 & **.26 & **.15 & .06 \\
\hline Multiple Choice & 7.41 & .01 & .13 & **.25 & ${ }^{* *} .15$ & .05 \\
\hline D-KEFS & $d f(1,33)$ & & & & & \\
\hline Free Response & 23.60 & $<.001$ & .42 & $* * .29$ & $* * .41$ & $* * .38$ \\
\hline Accuracy & 24.42 & $<.001$ & .43 & **.30 & **. 42 & $* * .40$ \\
\hline Abstract & 12.44 & .001 & .27 & $* .13$ & **.27 & **. 22 \\
\hline Multiple Choice & 1.48 & .23 & .04 & -- & -- & -- \\
\hline
\end{tabular}

Note: AgCC = participants with agenesis of corpus callosum; $\mathrm{VCI}=$ verbal comprehension index; FANL-C = Familiar and Novel Language Comprehension task. $P$ values for primary variables are adjust for multiple comparisons.

${ }^{* *} \mathrm{p}<.01 ;{ }^{*} \mathrm{p}<.05$.

For both free-response and multiple-choice, covarying verbal comprehension (VCI and age) increased the effect size of group differences. The group differences also remained for both free-response and multiple-choice when covarying literal language comprehension from the FANL-C, with essentially no change in effect size. However, the group differences for both free-response and multiple-choice were reduced to weak trends when controlling for non-literal scores on the FANL-C (freeresponse $\eta_{\mathrm{p}}{ }^{2}=.06, F(1,48)=2.87, p=.10$; multiple-choice $, \eta_{p}^{2}=.05, F(1,48)=2.29$, $p=.14)$. 


\subsection{Experiment 2: D-KEFS Proverbs}

Descriptive statistics for all D-KEFS Proverbs measures for each group are presented in Table 3 and results of univariate ANOVAs comparing groups are presented in Table 4. Separate univariate ANCOVAs were run to assess group differences in performance on the free-response and multiple-choice tasks using raw scores and age as a covariate. The AgCC group performed more poorly than the control group on the free-response total score, but the groups did not differ on multiple-choice total score. The multiple-choice data was not normally distributed, so it was also tested using Kruskal-Wallis test which was also not significant. Since no group differences were observed on the multiple-choice task, no additional analyses were warranted on these subtest scores.

As can be seen in Table 4, follow-up analyses of free-response sub-scores indicated that the AgCC group provided both less accurate and less abstract responses relative to the controls. The effect of the commonness of proverbs on group differences was tested using a repeated-measures ANOVA (group-bycommon vs. uncommon). There was, however, no overall effect for the commonness of proverbs, $\eta_{\mathrm{p}}^{2}=.02, F(1,33)=0.55, p=.46$, and no group-by-commonness interaction, $\eta_{\mathrm{p}}^{2}=.01, F(1,33)=0.46, p=.50$.

To better understand the overall group difference on the free-responses task, post-hoc analyses were conducted using additional covariates. The group difference remained when controlling for the effect of overall verbal comprehension skills (VCI), although the effect size decreased from $\eta_{\mathrm{p}}^{2}=.42$ to $\eta_{\mathrm{p}}{ }^{2}=.29$. When controlling 
for either literal or nonliteral subscales of the FANL-C, the group difference remained with minimal change in effect size.

Post-hoc analyses with the covariates were also conducted with respect to the accuracy and abstraction measures of the free-responses. The group effect for accuracy remained but with a reduced effect size (from $\eta_{p}^{2}=.43$ to $\eta_{p}{ }^{2}=.30$ ) when controlling for VCI, while group difference were minimally impacted by covarying literal and non-literal language comprehension as measured by the FANL-C. Likewise, group differences on abstraction scores from the free-response subtest remained significant with reduced effect size (from $\eta_{\mathrm{p}}{ }^{2}=.27$ to $\eta_{\mathrm{p}}{ }^{2}=.13$ ) when controlling for VCI, but controlling for literal and non-literal language comprehension had minimal impact on the group difference.

\section{Discussion}

Results of these studies support the hypothesis that callosal disconnection throughout development interferes with second-order language interpretation in adulthood. Specifically, our findings in the free response format of both tests provide further evidence that individuals with AgCC have reduced capacity to generate accurate interpretations of proverbs, and inconsistent outcomes across the two measures in the multiple choice format suggests that other factors may influence the degree to which individuals benefit from presentation of alternative interpretations, including differences in the nature of the proverbs used.

In the course of natural communication, listeners must be able to rapidly generate mental interpretations of formulaic language forms such as idioms, 
metaphors, and proverbs in order to comprehend a speaker's message. Although social communication does not typically require a listener to generate and orally present an interpretation, as is required in the free response tasks reported herein, it is nevertheless reasonable to say that the free response task offers an approximation of the cognitive requirements involved in naturalistic proverb interpretation. In providing alternatives from which to choose, the multiple choice format removes one fundamental requirement of proverb use in natural communication - the independent imagination of a meaning. Although questions remain regarding variation in multiple-choice performance in this research, our findings offer consistent and robust evidence that individuals with AgCC have impairments in proverb interpretation relevant to common social interaction.

\subsection{Free Response versus Multiple Choice}

While the results of the free-response versions of both proverbs tests were consistent in revealing a deficit in the performance of individuals with AgCC (Gorham, $\eta_{\mathrm{p}}^{2}=.13$; D-KEFS, $\eta_{\mathrm{p}}^{2}=.42$ ). Generally, the free responses given by individuals with $\mathrm{AgCC}$ were concrete restatements of the elements in the proverb. For example, in response to the proverb "Rome wasn't built in a day", one individual responded, "It took a long time to build the city"; or to the proverb, "A stream cannot rise higher than its source" the response was given "Water can't go any higher." However, on the multiple-choice tasks the AgCC group performed significantly more poorly than controls on the Gorham test, but not on the D-KEFS test (Gorham, $\eta_{p}{ }^{2}=$ .13; D-KEFS, $\left.\eta_{\mathrm{p}}{ }^{2}=.04\right)$. Thus, the current results on the Gorham Test are consistent with previously reported findings using this test in a smaller sample (Paul et al., 
2003) in that poorer performance was found in the AgCC group compared to controls on both response formats, with a non-significant group-by-format interaction (10 AgCC participants overlapped with the current sample).

In contrast, the current D-KEFS outcome (Experiment 2) was not consistent with either the Gorham outcome (Experiment 1), or with the previous findings: the AgCC group performed more poorly than controls on free-response format, but not on multiple-choice. Difference in outcome on the multiple choice tasks may be explained, at least in part, by task differences that are particularly salient for individuals with AgCC.

As described previously, because individuals with AgCC appear to have limited cognitive resources available for any given task, their performance is more markedly impacted by increased task novelty and complexity such as are evident in the Gorham test compared to the D-KEFS (Brown \& Paul, 2000; Paul et al., 2007). First, the Gorham multiple choice task includes many more items (40 versus 8 in the D-KEFS), and thus is more likely to include proverbs that are novel to the participant and complex to interpret. Novelty of D-KEFS multiple choice items is also reduced by using the same 8 items in both subtests. Thus, administration of the D-KEFS free response task provides participants with immediately preceding exposure to, and opportunity to consider, the meaning of all 8 proverbs prior to their presentation in the multiple-choice format. In contrast, the Gorham multiplechoice task presents the same 12 proverbs used in free response, but they are mixed into 28 entirely new proverbs. Second, there is a difference in the multiple choices that are available in the two tests. The D-KEFS choices follow a pattern of accurate- 
and-abstract (correct), accurate-and-concrete, related-but-not-relevant, and a nonsequitur. Gorham options do not offer a choice that is a non-sequitur. In addition, the Gorham choices offer one that is accurate and abstract (correct), two that are concrete, and one that is abstract but doesn't really address the whole proverb. So, the choices on the Gorham are also more challenging with respect to the finer discriminations that are needed to discern the best answer. Finally, the Gorham test was published in 1956 and includes some proverbs that are no longer commonly used. As such, the multiple-choice task would be more cognitively challenging to research participants, particularly those with AgCC.

\subsection{Parsing the Deficit in AgCC}

Post-hoc analysis of the sub-scores available in the D-KEFS Proverbs test allowed for additional investigation into outcomes with respect to accuracy, abstraction, and proverb familiarity. The DKEFS attempts to address the issue of proverb familiarity by providing sub-scores for common and uncommon proverbs. The common sub-score is comprised of five proverbs that the examinee is expected to be familiar with from past experience, whereas the uncommon score is based on three proverbs that are not as likely to have been encountered before. In the current study, this factor did not impact group differences. Both groups were similarly impacted by less common proverbs in the free response version, but commonness had no impact on either group in the multiple choice format. Nevertheless, having encountered the same proverbs earlier in the test itself (i.e., within the freeresponse version) may have contributed to normalizing the performance of individuals with AgCC in the multiple-choice version of the D-KEFS test. Given that 
the D-KEFS uses only 3 uncommon proverbs, the question regarding the impact of proverb familiarity remains uncertain. As argued above, the presence of a greater number of proverbs in the multiple choice version of the Gorham suggests that lack of familiarity with many of these proverbs may nevertheless have had some impact.

Sub-scores derived from the D-KEFS Proverbs test also provided the opportunity to isolate particular sources of difficulty. The abstraction subscale of the free-response format measures the ability of the examinee to generate an unaided response that is sufficiently abstract to have broad situational applicability. A high score on accuracy with a low score on abstraction suggests adequate understanding of the basic semantic relationships between the elements of the proverb, but a deficiency in the ability to comprehend and/or use language beyond a concrete level (Delis et al., 2001). Significantly low scores in both accuracy and abstraction suggest difficulty in comprehending both basic semantic relationships and in generating abstract (second-order) interpretations of proverbs. In the current study, both the accuracy and the abstraction scores showed robust group differences $\left(\eta_{p}^{2}=.43\right.$ and $\eta_{p}^{2}=.27$, respectively), albeit based on a small number of proverbs.

To address the possibility that basic language skills may account for group differences in proverb interpretation, comparisons of $\mathrm{AgCC}$ and control groups were reanalyzed with addition of two measures of basic language capacities - VCI and scores on the comprehension of literal language statements from the FANL-C. Covarying VCI increased effect size of group differences on both formats of the Gorham Proverb test by $\sim 100 \%$, indicating that group differences in proverb 
comprehension were actually obscured to some degree by variance created by basic verbal capacities. However, on the D-KEFS free-response, covarying VCI actually decreased the effect size by $31 \%$ but the group difference remained significant (Table 4). Variance related to basic literal language comprehension on the FANL-C did not account for the group differences in proverb interpretation on either task. Thus, it appears that impaired proverb comprehension in AgCC is not simply a consequence of impairment in more general verbal skills or literal language comprehension.

In a previous study of humor comprehension in individuals with AgCC (Brown, Paul, et al., 2005) we found that group differences in humor were markedly diminished when covarying either scores on the non-literal subtest of the FANL-C or scores on the Gorham Proverbs test - that is, deficiencies in the comprehension of humor, proverbs, and non-literal language were highly correlated. We interpreted these findings as suggesting that individuals with AgCC have difficulty with the comprehension of the second-order meanings of statements as demanded by all three tests.

In the current studies, the AgCC group had significantly lower scores than the controls on the non-literal subtest of the FANL-C (Experiment $1 \eta^{2} p=.23$; Experiment $2 \eta^{2}{ }^{2}=.08$ ), consistent with results reported previously (Paul et al., 2003). Covarying this score in Experiment 1 reduced the effect size for both the free response and multiple choice tests by half, and eliminated the significant difference between groups (Table 4). In contrast, covarying FANL-C non-literal language scores had minimal impact on the significant group difference for D-KEFS free- 
response scores (Table 4). This suggests that group differences on the Gorham are closely related to skills in non-literal language comprehension, but non-literal language comprehension does not account for difference on D-KEFS proverb interpretation. This outcome supports the notion that there is some difference between these proverb interpretation tests in their cognitive demands. Perhaps more familiar proverbs have meanings available on a more rote and immediate basis, whereas less familiar proverbs (more likely to be encountered in the older Gorham test, particularly with the large number of items in the multiple choice test) would require generation of novel, on-the-spot non-literal language interpretations - more similar to the cognitive demands created by humor, for example. Nippold and Rudzinski (1993) argue that metaphorical language that has been conventionalized (idioms and common proverbs) is stored in memory as a whole, with associated meanings, whereas unfamiliar metaphorical language (particularly uncommon proverbs) requires the reader to derive meaning from individual words and context and therefore utilize more cognitive resources.

\subsection{Callosal Function and Proverb Comprehension}

There are two perspectives from which to explain deficient performance in proverb interpretation in individuals with $\mathrm{AgCC}$ on these proverbs tasks, and thus to understand the contribution of the corpus callosum to this capacity: hemispheric specialization and processing resource limitations. These are complimentary perspectives on this relationship, not contradictory alternatives.

From the point of view of hemispheric specialization, the theory is that absence of the corpus callosum disconnects hemispherically lateralized processors 
that contribute to proverb interpretation. The dominant role of the left hemisphere in language has been relatively clear for many decades, while more recent research highlights right-hemisphere contributions to semantic and paralinguistic aspects of language (Van Lanker Sidtis \& Postman, 2006).

The role of the RH in non-literal language comprehension is likely subtle (Van Lancker Sidtis, 2006). Various studies have shown that individuals with rightor left-hemisphere damage may achieve similar scores on particular neuropsychological tasks but use distinctive strategies to achieve the end goal (Bever, 1975; Bogen, 1969; Martin, 1979; van Lancker \& Sidtis, 1992). The left hemisphere may be able to do most of the work that is involved in comprehending and generating verbal information, but it seems clear that the right hemisphere plays an essential role in a variety of forms of verbal communication, not least of which involves processing non-literal language (Joanette, Goulet, Hannequin, \& Boeglin, 1989; Myers, 1998). For example, patients with left-hemisphere damage may have preserved use of formulaic language, including proverbs (Geschwind, Quadfase, \& Segarra, 1968; Nakagawa et al., 1993); while a variety of forms of formulaic language have been found to be impaired in right-hemisphere damaged patients including idioms (Bryan, 1988; Burgess \& Chiarello, 1996), metaphors (Winner \& Gardner, 1977), indirect requests (Weylman, Brownell, Roman, \& Gardner, 1989), and conversations (Rehak, Kaplan, \& Gardner, 1992). Individuals with right-hemisphere damage may also have deficits in other language-relevant capacities such as comprehending emotional meanings, pragmatic communication, and emotion in music (Gardner, Silverman, Denes, Semenza, \& Rosenstiel, 1977; 
Kaplan, Brownell, Jacobs, \& Gardner, 1990; Rehak et al., 1992; Winner \& Gardner, 1977).

In light of these findings, we might infer that interhemispheric transfer limitations in AgCC decrease availability of the paralinguistic information processing of the right hemisphere to the primary language areas of the left hemisphere (Paul et al., 2003).

From the perspective processing resources, the theory here focuses on the role of the corpus callosum in marshaling large neural networks to process information of all sorts. As information processing becomes more complex and novel, there is greater requirement for integrating activity of multiple neural networks and the contribution of callosal connectivity gets more significant. Thus, lack of callosal interconnectivity in individuals with AgCC would reduce the availability of large-scale interhemispheric networks that might otherwise be important in efficiently and quickly addressing particular novel and complex problems. While primary language processing, once it becomes habitual, might be done in a single hemisphere, contextually dependent alternative meanings or novel semantic forms (such as non-literal language, humor, and proverbs) might require a large bi-hemispheric network for rapid and efficient processing to occur. This perspective also suggests why the free-response versions of the proverbs tests (more complex) result in greater deficits among individuals with AgCC than do the multiple-choice version of the D-KEFS (less complex - and reduced novelty in the case of the D-KEFS). Consistent with this notion, Van Lancker Sidtis (2006) argued 
that it takes the whole brain to comprehend and generate non-literal language efficiently.

\section{Conclusions}

There are several limitations to this research worth noting. Comparisons between the multiple-choice versions of the Gorham and D-KEFS did not allow genuine replication since the multiple-choice of the D-KEFS reuses proverbs given in the free-response version. In addition, the subscales of the D-KEFS include a limited number of proverbs and thus some indices are calculated from a small number of items. The free-response format of the D-KEFS Proverbs test includes just eight proverbs, and only three of those fall into the uncommon proverbs category. Thus, the common-uncommon distinction in this research is based on too few items for the finding to be considered highly robust. Finally, since proverbs are culturallydependent, it is possible that items in the Gorham test are more likely to be unfamiliar to current research participants than items from the more recently developed D-KEFS.

Despite these limitations, the results of this study clearly show that proverb comprehension is diminished in individuals with AgCC compared to their peers. The difference between groups was greatest when encountering proverbs for the first time on the test (i.e., both forms of the Gorham test, and the free response format of the D-KEFS). Further work is necessary in order to characterize the impact that other cognitive impairments outside the language domain in AgCC, particularly in executive skills and generative/imaginative capacities, may have on comprehension 
of second-order meanings of proverbs and other forms of non-literal language.

Social interactions in daily life pose significant challenges for persons with AgCC. The current findings describe one factor which may contribute to such challenges, a deficit in accurate, spontaneous interpretation of proverbs. Proverbs are one of many linguistic forms that require comprehension of second-order meanings; thus, while they are not the only language structure which pose a challenge to people with AgCC, they are particularly relevant to clarifying misunderstandings these individuals may have in daily communication. 


\section{Acknowledgements}

This paper is derived from the doctoral dissertation of Jamie L. Rehmel at the Fuller Graduate School of Psychology. It is part of a long-term larger research project on Cognitive and Psychosocial Deficits in Agenesis of the Corpus Callosum, and is thus indebted to many who have carried on this project over more than twenty years of research. 


\section{References}

Bever, T. G. (1975). Cerebral asymmetries in humans are due to the differentiation of two incompatible processes: holistic and analytic. Ann N Y Acad Sci, 263, 251-262.

Bihrle, A. M., Brownell, H. H., Powelson, J. A., \& Gardner, H. (1986). Comprehension of humorous and nonhumorous materials by left and right brain-damaged patients. Brain Cogn, 5(4), 399-411.

Bogen, J. E. (1969). The other side of the brain. II. An appositional mind. Bull Los Angeles Neurol Soc, 34(3), 135-162.

Brown, W., \& Paul, L. (2000). Cognitive and psychosocial deficits in agenesis of the corpus callosum with normal intelligence. Cognitive neuropsychiatry, 5(2), 135-157.

Brown, W. S., Jeeves, M. A., Dietrich, R., \& Burnison, D. S. (1999). Bilateral field advantage and evoked potential interhemispheric transmission in commissurotomy and callosal agenesis. Neuropsychologia, 37(10), 11651180.

Brown, W. S., \& Paul, L. K. (2000). Cognitive and psychosocial deficits in agenesis of the corpus callosum with normal intelligence. Cognitive and psychosocial deficits in agenesis of the corpus callosum with normal intelligence. doi:10.1080/135468000395781

Brown, W. S., Paul, L. K., Symington, M., \& Dietrich, R. (2005). Comprehension of humor in primary agenesis of the corpus callosum. Neuropsychologia, 43(6), 906-916. doi:10.1016/j.neuropsychologia.2004.09.008

Brown, W. S., Symingtion, M., VanLancker-Sidtis, D., Dietrich, R., \& Paul, L. K. (2005). Paralinguistic processing in children with callosal agenesis: emergence of neurolinguistic deficits. Brain and Language, 93(2), 135-139. doi:10.1016/j.bandl.2004.09.003

Brown, W. S., Thrasher, E. D., \& Paul, L. K. (2001). Interhemispheric Stroop effects in partial and complete agenesis of the corpus callosum.J Int Neuropsychol Soc, 7(3), 302-311.

Brune, M., \& Bodenstein, L. (2005). Proverb comprehension reconsidered--'theory of mind' and the pragmatic use of language in schizophrenia. Schizophr Res, 75(2-3), 233-239. doi:10.1016/j.schres.2004.11.006

Bryan, K. L. (1988). Assessment of language disorders after right hemisphere damage. Br J Disord Commun, 23(2), 111-125.

Burgess, C., \& Chiarello, C. (1996). Neurocognitive mechanisms underlying metaphor comprehension and other figurative language. Metaphor and Symbolic Activity, 11, 67 - 84. doi:10.1207/s15327868ms1101_4

Chiarello, C. (1980). A house divided? Cognitive functioning with callosal agenesis. Brain and Language, 11, 128-158.

Coulson, S., \& Williams, R. F. (2005). Hemispheric asymmetries and joke comprehension. Neuropsychologia, 43(1), 128-141.

doi:10.1016/j.neuropsychologia.2004.03.015 
Delis, D. C., Kaplan, E., \& Kramer, J. H. (2001). The Delis-Kaplan Executive Function System: Technical Manual. San Antonio, TX: The Psychological Corporation.

Erickson, R. L., Paul, L. K., \& Brown, W. S. (2014). Verbal learning and memory in agenesis of the corpus callosum. Neuropsychologia, 60, 121-130. doi:10.1016/j.neuropsychologia.2014.06.003

Gardner, H., Silverman, J., Denes, G., Semenza, C., \& Rosenstiel, A. K. (1977). Sensitivity to musical denotation and connotation in organic patients. Cortex, 13(3), 242-256.

Geschwind, N., Quadfase, F., \& Segarra, J. M. (1968). Isolation of Speech Area. Neuropsychologia, 6(4), 327-\&. doi:Doi 10.1016/0028-3932(68)90005-5

Gorham, D. R. (1956). Use of the proverbs test for differentiating schizophrenics from normals. J Consult Psychol, 20(6), 435-440.

Gott, P. S., \& Saul, R. E. (1978). Agenesis of the corpus callosum: limits of functional compensation. Neurology, 28(12), 1272-1279.

Hines, R., Paul, L., \& Brown, W. (2002). Spatial attention in agenesis of the corpus callosum: shifting attention between visual fields. Neuropsychologia, 40(11), 1804-1814.

Imamura, T., Yamadori, A., Shiga, Y., Sahara, M., \& Abiko, H. (1994). Is distrurbed transfer of learning in callosal agenesis due to a disconnection syndrome? Behavioural Neurology, 7, 43-48.

Jeeves, M. A. (1979). Some limits to interhemispheric integration in cases of callosal agenesis and partial commissurotomy. In I. S. Russell, M. W. Van Hof, \& G. Berlucci (Eds.), Structure and function of the cerebral commissures (pp. 449 474). London: McMillan.

Jeeves, M. A., \& Silver, P. H. (1988). Interhemispheric transfer of spatial tactile information in callosal agenesis and partial commissurotomy. Cortex, 24, 601-604.

Jeeves, M. A., Silver, P. H., \& Jacobson, I. (1988). Bimanual co-ordination in callosal agenesis and partial commissurotomy. Neuropsychologia, 26, 833-850.

Jeeves, M. A., Silver, P. H., \& Milner, A. D. (1988). Role of the corpus callosum in the development of a bimanual skills. Developmental neuropsychology, 4, 305323.

Joanette, Y., Goulet, P., Hannequin, D., \& Boeglin, J. (1989). Right hemisphere and verbal communication. Berlin: Springer.

Kaiser, N. C., Lee, G. J., Lu, P. H., Mather, M. J., Shapira, J., Jimenez, E., ... Mendez, M. F. (2013). What dementia reveals about proverb interpretation and its neuroanatomical correlates. Neuropsychologia, 51(9), 1726-1733. doi:10.1016/j.neuropsychologia.2013.05.021

Kaplan, J., Brownell, H., Jacobs, J., \& Gardner, H. (1990). The effects of right hemisphere damage on the pragmatic interpretation of conversational remarks. Brain and Language, 38, 315-333.

Karnath, H. O., Schumacher, M., \& Wallesch, C. W. (1991). Limitations of interhemispheric extracallosal transfer of visual information in callosal agenesis. Cortex, 27, 345-350.

Kempler, D., \& Van Lancker-Sidtis, D. (1996). Formulaic and novel language comprehension test. 
Kiang, M., Light, G. A., Prugh, J., Coulson, S., Braff, D. L., \& Kutas, M. (2007). Cognitive, neurophysiological, and functional correlates of proverb interpretation abnormalities in schizophrenia. J Int Neuropsychol Soc, 13(4), 653-663. doi:10.1017/S1355617707070816

Marco, E. J., Harrell, K. M., Brown, W. S., Hill, S. S., Jeremy, R. J., Kramer, J. H., . . Paul, L. K. (2012). Processing speed delays contribute to executive function deficits in individuals with agenesis of the corpus callosum. J Int Neuropsychol Soc, 18(3), 521-529. doi:10.1017/S1355617712000045

Martin, M. (1979). Hemispheric specialization for local and global processing. Neuropsychologia, 17(1), 33-40.

McDonald, C. R., Delis, D. C., Kramer, J. H., Tecoma, E. S., \& Iragui, V. J. (2008). A componential analysis of proverb interpretation in patients with frontal lobe epilepsy and temporal lobe epilepsy: relationships with disease-related factors. Clin Neuropsychol, 22(3), 480-496. doi:10.1080/13854040701363828

Mieder, Wolfgang (1993). Proverbs are never out of season: Popular wisdom in the modern age. Oxford, U.K.: Oxford University Press.

Moran, C. A., Nippold, M. A., \& Gillon, G. T. (2006). Working memory and proverb comprehension in adolescents with traumatic brain injury: a preliminary investigation. Brain Inj, 20(4), 417-423. doi:10.1080/02699050500488223

Mueller, K. L. O., Marion, S. D., Paul, L. K., \& Brown, W. S. (2009). Bimanual Motor Coordination in Agenesis of the Corpus Callosum. Behavioral Neuroscience, 123, 1000-1011.

Murphy, P., Shallice, T., Robinson, G., MacPherson, S. E., Turner, M., Woollett, K., .. . Cipolotti, L. (2013). Impairments in proverb interpretation following focal frontal lobe lesions. Neuropsychologia, 51(11), 2075-2086. doi:10.1016/j.neuropsychologia.2013.06.029

Myers, P. (1998). Right hemisphere damage: Disorders of communication and cognition. San Diego, CA: Singular.

Nakagawa, Y., Tanabe, H., Ikeda, M., Kazui, H., Ito, K., Inoue, N., . . Shiraishi, J. (1993). Completion phenomenon in transcortical sensory aphasia. Behav Neurol, 6(3), 135-142. doi:10.3233/BEN-1993-6304

Nippold, M. A., Allen, M. M., \& Kirsch, D. I. (2000). How adolescents comprehend unfamiliar proverbs: the role of top-down and bottom-up processes. J Speech Lang Hear Res, 43(3), 621-630.

Nippold, M. A., Allen, M. M., \& Kirsch, D. I. (2001). Proverb comprehension as a function of reading comprehension in preadolescents. Lang Speech Hear Serv Sch, 32, 621 - 630. doi:10.1044/jslhr.4303.621

Nippold, M. A., \& Sullivan, M. P. (1987). Verbal and perceptual analogical reasoning and proportional metaphor comprehension in young children.J Speech Hear Res, 30(3), 367-376.

Nippold, M. A., Uhden, L. D., \& Schwarz, I. E. (1997). Proverb explanation through the lifespan: a developmental study of adolescents and adults. J Speech Lang Hear Res, 40(2), 245-253.

Nippold, M. A., \& Rudzinski, M. (1993). Familiarity and transparency in idiom explanation: A developmental study of children and adolescents. Journal of 
Speech and Hearing Research, 36, 728-737. doi:10.1044/jshr.3604.728

Oldfield, R.C.(1971).The assessment and analysis of handedness: The Edinburgh inventory. Neuropsychologia, 9, 97-113.

Paul, L. (2004). Social processing deficits in agenesis of the corpus callosum: narratives from the Thematic Apperception Test. Archives of Clinical Neuropsychology, 19. doi:10.1016/S0887-6177(03)00024-6

Paul, L. K. (2011). Developmental malformation of the corpus callosum: a review of typical callosal development and examples of developmental disorders with callosal involvement. J Neurodev Disord, 3(1), 3-27. doi:10.1007/s11689-0109059-y

Paul, L. K., Brown, W. S., Adolphs, R., Tyszka, J. M., Richards, L. J., Mukherjee, P., \& Sherr, E. H. (2007). Agenesis of the corpus callosum: genetic, developmental and functional aspects of connectivity. Nat Rev Neurosci, 8(4), 287-299. doi: $10.1038 / \mathrm{nrn} 2107$

Paul, L. K., Van Lancker-Sidtis, D., Schieffer, B., Dietrich, R., \& Brown, W. S. (2003). Communicative deficits in agenesis of the corpus callosum: nonliteral language and affective prosody. Brain and Language, 85(2), 313-324.

Rauch, R. A., \& Jinkins, J. R. (1994). Magnetic Resonance Imaging of Corpus Callosum Dysgensis. In M. Lassonde \& M. A. Jeeves (Eds.), Callosal Agenesis: A Natural Split Brain? (pp. 83-95). New York: Plenum Press.

Rehak, A., Kaplan, J. A., \& Gardner, H. (1992). Sensitivity to conversational deviance in right-hemisphere-damaged patients. Brain Lang, 42(2), 203-217.

Roca, M., Parr, A., Thompson, R., Woolgar, A., Torralva, T., Antoun, N., ... Duncan, J. (2010). Executive function and fluid intelligence after frontal lobe lesions. Brain, 133(Pt 1), 234-247. doi:10.1093/brain/awp269

Sauerwein, H. C., \& Lassonde, M. (1983). Intra- and inter-hemispheric processing of visual information in callosal agenesis. Neuropsychologia, 21, 167-171.

Sauerwein, H. C., Nolin, P., \& Lassonde, M. (1994). Cognitive functioning in callosal agenesis. In M. Lassonde \& M. A. Jeeves (Eds.), Callosal agenesis: A natural split brain? (pp. 221-233). New York: Plenum Press.

Smith, L., Rourke, B., \& Rourke, B. P. (1994). Callosal agenesis: a case study in NLD.

Solursh, L. P., Margulies, A. I., Ashem, B., \& Stasiak, E. A. (1965). The relationships of agenesis of the corpus callosum to perception and learning. J Nerv Ment Dis, 141(2), 180-189.

Symington, S. H., Paul, L. K., Symington, M. F., Ono, M., \& Brown, W. S. (2010). Social cognition in individuals with agenesis of the corpus callosum. Soc Neurosci, 5(3), 296-308. doi:10.1080/17470910903462419

Turk, A., Brown, W. S., Symingtion, M., \& Paul, L. K. (2010). Social narratives in agenesis of the corpus callosum: Linguistic analysis of the Thematic Apperception Test. Neuropsychologia, 48, 43-50.

Uekermann, J., Thoma, P., \& Daum, I. (2008). Proverb interpretation changes in aging. Brain Cogn, 67(1), 51-57. doi:10.1016/j.bandc.2007.11.003

van Lancker, D., \& Sidtis, J. (1992). The identification of affective-prosodic stimuli by left- and right-hemisphere-damaged subjects: all errors are not created equal. Journal of Speech and Hearing Research, 35, 963-970. 
Van Lancker Sidtis, D. (2006). Where in the brain is nonliteral language? . Metaphor and Symbol, 21, 213 - 244. doi:10.1207/s15327868ms2104_2

Van Lanker Sidtis, D., \& Postman, W. A. (2006). Formulaic expressions in spontaneous speech of left hemisphere- and right hemisphere-damaged subjects. Aphasiology, 20, 411 - 426. doi:10.1080/02687030500538148

Wechsler, D. (1997). Wechsler Adult Intelligence Scale - Third Edition. San Antonio, Tx: The Psychological Corporation.

Weylman, S., Brownell, H., Roman, M., \& Gardner, H. (1989). Appreciation of indirect requests by left and right brain-damaged patients: the effects of verbal context and conventionality of wording. Brain and Language, 36, 580-591.

Wilde, E. A., Chu, Z., Bigler, E. D., Hunter, J. V., Fearing, M. A., Hanten, G., . . Levin, H. S. (2006). Diffusion tensor imaging in the corpus callosum in children after moderate to severe traumatic brain injury.J Neurotrauma, 23(10), 14121426. doi:10.1089/neu.2006.23.1412

Winner, E., \& Gardner, H. (1977). The comprehension of metaphor in brain-damaged patients. Brain, 100(4), 717-729.

Yang, F. G., Fuller, J., Khodaparast, N., \& Krawczyk, D. C. (2010). Figurative language processing after traumatic brain injury in adults: a preliminary study. Neuropsychologia, 48(7), 1923-1929. doi:10.1016/j.neuropsychologia.2010.03.011 